\title{
ORIGINAL RESEARCH \\ Placement of Covered Stents for the Treatment of Direct Carotid Cavernous Fistulas
}

C. Wang

X. Xie

C. You

C. Zhang

M. Cheng

M. He

H. Sun

B. Mao

BACKGROUND AND PURPOSE: Endovascular detachable balloon occlusion and coil occlusion are 2 well-established options for the treatment of direct carotid cavernous fistulas (DCCFs). In recent years, covered stents have been applied in the treatment of neurovascular pathologies such as aneurysms, pseudoaneurysms, arterial dissections, and DCCFs. The purpose of this study was to investigate the clinical efficacy of covered stents for the treatment of DCCFs.

MATERIALS AND METHODS: Ten consecutive patients underwent covered-stent placement after failure of detachable balloon occlusion for the treatment of their DCCFs. Clinical and angiographic follow-up ranged from 5 to 48 months (mean, 18.2 months) after stent placement.

RESULTS: Stent placement was technically successful in all except 1 patient. In this patient, stent placement failed after multiple attempts because of rigidity of the Jostent GraftMaster Coronary Stent Graft and the tortuous anatomy of the internal carotid artery (ICA). Complete exclusion of the fistula was achieved in 6 patients immediately after stent deployment. Endoleak was observed in 3 patients. Re-dilation of the stent avoided the endoleak in 2 patients; in 1 of these 2 patients, formerly improved symptoms recurred the next morning and the ipsilateral ICA was occluded with detachable balloons. Spasm of the ICA was observed in most of the patients after stent placement; however, angioplasty was not required. Symptoms improved in all patients after treatment, without thromboembolic events. Follow-up cerebral angiography showed complete exclusion of all DCCFs and stent patency without intrastent stenosis in the 8 patients who had successful deployment of the stent.

CONCLUSIONS: Although a larger sample and expanded follow-up are needed, our series shows that covered stents can be used in the treatment of DCCFs with symptomatic relief

D irect carotid cavernous fistulas (DCCFs) represent a direct communication between the internal carotid artery (ICA) and the cavernous sinus, classified by Barrow et $\mathrm{al}^{1}$ as a type $\mathrm{A}$ fistula. Most DCCFs are traumatic; however, spontaneous fistulas arise from rupture of carotid cavernous aneurysms thus allowing communication with the cavernous sinus. ${ }^{2-4}$ Patients having DCCFs clinically present with the following symptoms: exophthalmos, conjunctival injection, bruit, and cranial nerve impairment. Treatment of DCCFs includes endovascular methods of transarterial or transvenous embolization or an open approach with superior ophthalmic vein exposure. ${ }^{2-4}$ In recent years, covered stents have been applied for the treatment of DCCFs with successful short-to-midterm clinical results. ${ }^{2,5-8}$ We report 10 cases of DCCFs treated with covered stents after failure of detachable balloon occlusion.

\section{Materials and Methods}

\section{Patients}

Between October 2003 and October 2007, 10 consecutive patients underwent placement of a covered stent (Jostent GraftMaster Coronary Stent Graft; Abbott Vascular, Redwood City, Calif) for the treatment of their DCCFs. We retrospectively reviewed their medical records with approval of the institutional review board at West China Hospital, Sichuan University.

Received December 23, 2008; accepted after revision February 3, 2009

From the Department of Neurosurgery, West China Hospital, Sichuan University, Chengdu, P.R. China.

Please address correspondence to Xiaodong Xie, MD, or Chaohua Wang, MD, Department of Neurosurgery, West China Hospital, Sichuan University, Chengdu, P.R. China, 610041; e-mail: xiaodong_1962@163.com or chaohuawang@sina.com

三E indicates article with supplemental on-line table.

DOI 10.3174/ajnr.A1583
There were 4 men and 6 women (24-68 years of age; mean, 50 years). Seven cases were traumatic and 3 were spontaneous. Patients had clinical manifestation consisting of pulsatile exophthalmos, conjunctival injection, bruit, and cranial nerve impairment (on-line Table).

All patients underwent a well-tolerated temporary balloon occlusion (TBO) test and initial treatment with detachable balloon occlusion. In 5 patients (cases 1, 2, 4, 8, and 9), the fistula continued to be patent after detachment of $2-5$ balloons. Additional balloons posed a greater risk than opportunity for complete occlusion. In cases 3 and 6 , the fistula recurred due to early balloon deflation as evident on angiography at 2 and 5 weeks after treatment. Both cases 5 and 7 experienced 2 unsuccessful procedures during which neither a balloon nor a microcatheter could be navigated into the fistula. In case 10, the balloon ruptured on mild inflation within the fistula.

In all of the above-mentioned patients, informed consent was obtained from the patients or their legal representatives for the treatment of the fistulas with a covered stent. Patients and family members were informed of the treatment options: transarterial or transvenous coil embolization, sacrifice of the ipsilateral ICA, and Jostent GraftMaster Coronary Stent Graft. Patients and family members understood that the use of the Jostent GraftMaster Coronary Stent Graft was experimental and that its design was not specifically for intracranial use.

\section{Methods}

All patients were premedicated with aspirin $(150 \mathrm{mg} /$ day $)$ and clopidogrel ( $75 \mathrm{mg} /$ day) orally at least 3 days before covered-stent placement. With the patient under local anesthesia, an $8 \mathrm{~F}-10 \mathrm{~F}$ introducer sheath (Cordis, Miami Lakes, Fla) was placed in the right or left femoral artery by using the Seldinger technique. Patients were heparinized during the procedure with the goal of an activated clotting time of twice that of the baseline. Using an $8 \mathrm{~F}$ guiding catheter (Cordis), the 

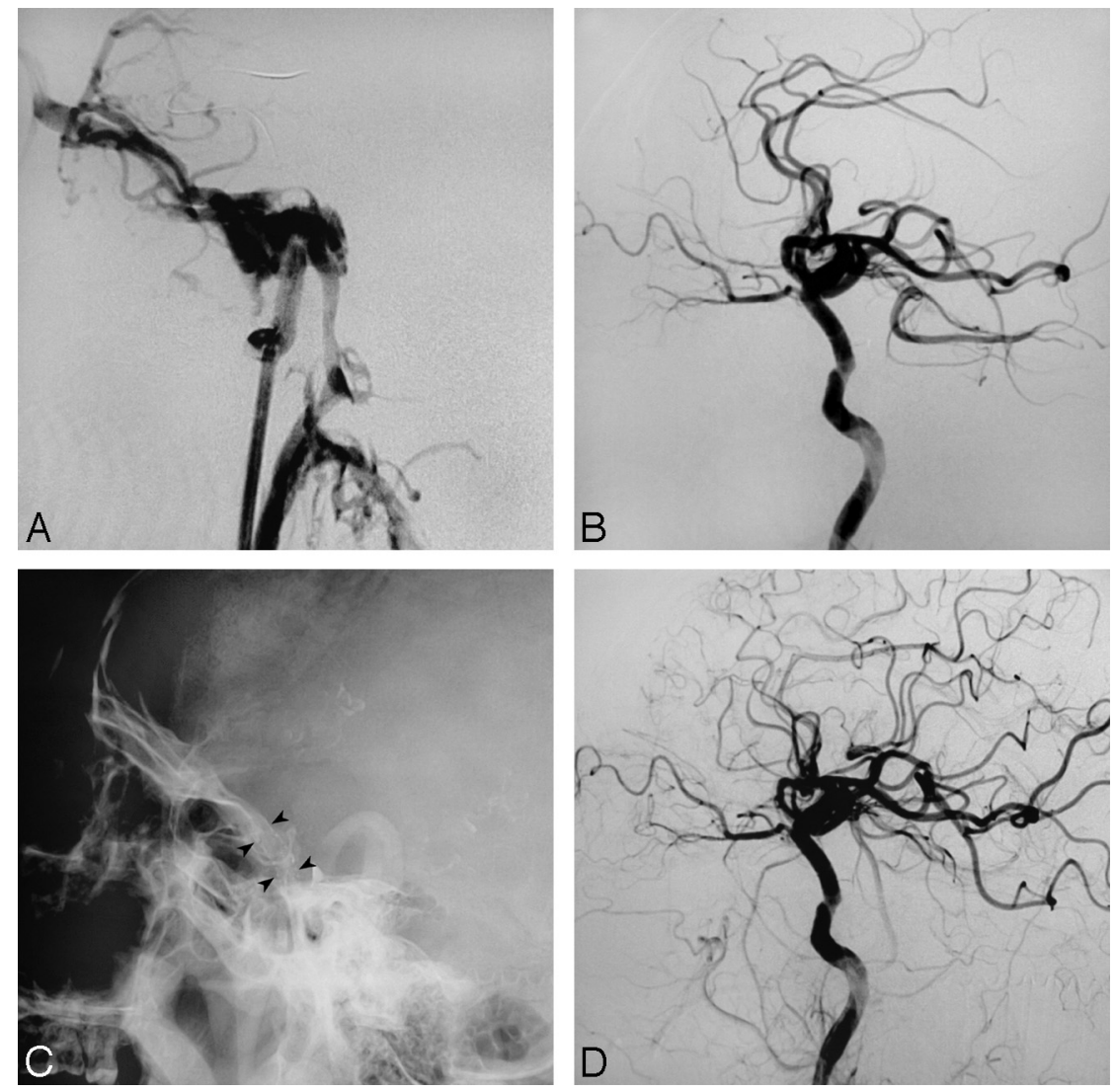

Fig 1. Case 5. A, Left carotid angiogram shows a DCCF. B, Immediate angiogram after covered-stent deployment shows complete occlusion of the fistula. Note the rigid course of the ICA where the stent has been deployed. $C$, Fluoroscopy shows the stent (arrowheads). D, Follow-up angiogram at 5 months shows no recanalization of the fistula and patency of the stent.

ipsilateral ICA was catheterized and cerebral angiography was performed to evaluate the angioarchitecture of the fistula. Next, a $0.014-$ inch exchange mircoguidewire $(300 \mathrm{~cm}$ in length, Transend Floppy; Boston Scientific, Natick, Mass) was navigated into a distal branch of the middle cerebral artery (MCA) or the anterior cerebral artery. Then a Jostent GraftMaster Coronary Stent Graft of appropriate diameter was placed over the wire under roadmap guidance to the diseased segment of the ICA. Multiple control angiograms were obtained to confirm the correct position. Then the stent was inflated with 8-10 atm of pressure.

Cerebral angiography was performed after deflation and withdrawal of the balloon. If an endoleak was revealed, re-dilation was performed with the balloon of the stent graft by a higher pressure up to $12-16 \mathrm{~atm}$ or with a balloon of a larger diameter $(5.0-6.0 \mathrm{~mm}$ in diameter, Amiia; Cordis, Warren, NJ). After the procedure, low-molecular-weight heparin was given subcutaneously every 12 hours for 72 hours. Patients were instructed to take aspirin $(150 \mathrm{mg} /$ day $)$ and clopidogrel (75 mg/day) orally. Clopidogrel was continued for 12 weeks, whereas aspirin was continued indefinitely.

\section{Results}

Stent deployment was technically successful in 9 of 10 patients. In case 2 , the stent could not be navigated to the diseased segment because of rigidity of the stent and tortuosity of the carotid artery. After trying a long sheath and coaxial double-guiding systems ( $8 \mathrm{~F}$ and $10 \mathrm{~F}$ ), we were still unable to guide the stent delivery system to the diseased segment of the ICA. This patient was then embolized with coils via the direct superior ophthalmic vein approach.
Complete exclusion of the fistula was achieved in 6 patients immediately after stent deployment (Fig 1). Endoleak was observed in 3 patients (cases 3, 6, and 7), thus re-dilation of the stent with a balloon of a larger diameter was performed, which avoided the endoleak in 2 patients (cases 3 and 7 ) (Fig 2). In case 6, there was slow and sluggish filling of the cavernous sinus after re-dilation; however, the fistula was still patent. Symptoms improved dramatically within several hours in case 7 but recurred the next morning. Fluoroscopy showed the stent in the original place but cerebral angiography revealed a large endoleak and the fistula persisted to be high flow. The ipsilateral ICA was occluded with detachable balloons after a successful balloon-occlusion test (Fig 3).

Spasm of the ICA was observed in most of the patients after stent deployment and was mainly appreciated at both ends of the stent, manifesting as mild-to-moderate local stenosis. The angiographically evident spasm was clinically silent and thus did not require treatment.

All cases were followed clinically and angiographically. Follow-up ranged from 5 to 48 months, with a mean of 18.2 months. Clinically, all patients improved without any evidence of clinically significant thromboembolic events. Cerebral angiography revealed complete occlusion of the DCCFs in all patients, including case 6, in whom the DCCF was patent after treatment. In addition, cerebral angiography revealed patency of the stent without intrastent stenosis. 

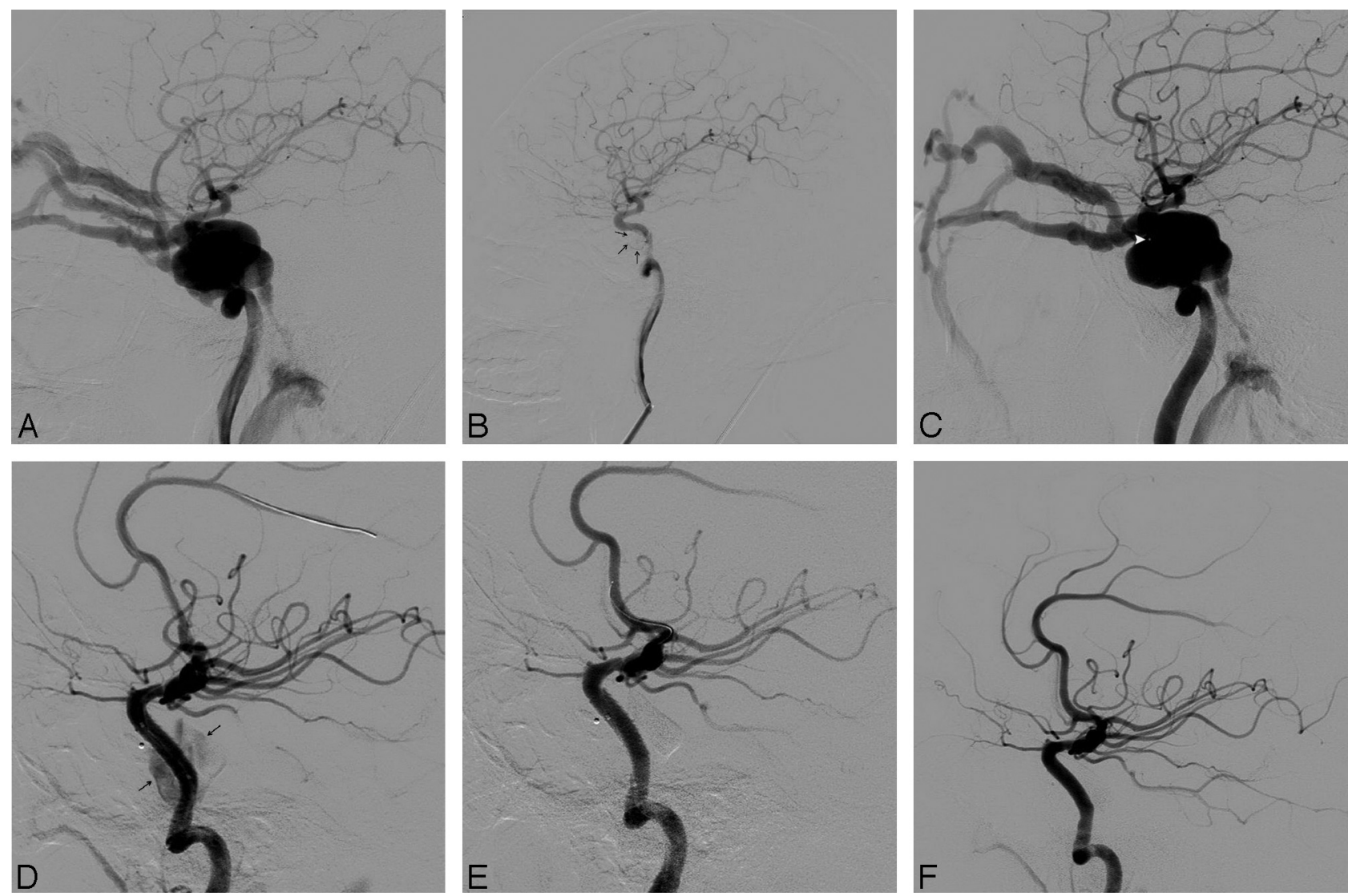

Fig 2. Case 3. $A$, Left carotid angiogram shows a DCCF. $B$, Immediate angiogram after balloon (arrows) detachment shows complete occlusion of the fistula. $C$, The fistula recurred due to early balloon deflation as evident on angiography at 2 weeks after balloon detachment. The small white point (arrowhead) is the marker of the balloon. $D$, Immediate angiogram after covered-stent deployment shows sluggish filling of the cavernous sinus (arrows). E, Angiogram shows complete occlusion of the fistula after re-dilation. F, Follow-up angiogram at 48 months shows no recanalization of the fistula and patency of the stent.

\section{Discussion}

In 1973, Parkinson ${ }^{9}$ reported successful direct surgical repair of DCCFs with preservation of the parent artery, but the technical difficulty and significant morbidity of this procedure have precluded its widespread use. Since Serbinenko ${ }^{10}$ reported his experience with a detachable balloon, endovascular treatment has become the primary treatment technique for DCCFs.

Most authors prefer detachable balloon occlusion because it is technically easier. A minimally inflated balloon can be easily navigated into the fistula by flow guidance. When necessary, the balloon can be deflated and repositioned before detachment without difficulty. In addition to being userfriendly, balloons are cheap in a highly expensive medical market. ${ }^{11,12}$

Balloons do have their pitfalls. The fistula and the cavernous sinus must be of a proper size. A balloon cannot be navigated into a small fistula. In addition, a balloon may protrude and even migrate into the ICA if the size of fistula is too large and that of the cavernous sinus is too small. Large balloons or multiple balloons occupying the cavernous sinus can cause cranial nerve palsies or can delay recovery from pre-existing palsies from the inherent mass effect of the balloons. ${ }^{11-14}$ In addition, premature deflation of the balloon leads to recurrence of the fistula/aneurysm or formation of a pseudoaneurysm. ${ }^{3,4,12,13}$

Transarterial or transvenous detachable coil embolization is an effective alternative for the treatment of DCCFs. Detachable coils are easy to retrieve, reposition, or exchange when necessary. They may also protrude or migrate into the ICA, but modified techniques by using a bare stent or balloon-catheter technology prevent such risks. ${ }^{3,15}$ Nonetheless coils have disadvantages similar to those of balloons: mass effect and recurrence of the fistula/aneurysm through coil compaction. . $^{3,4}$

Luo et $\mathrm{al}^{16}$ reported their experience of $n$-butyl 2-cyanoacrylate for the treatment of DCCFs with good results and without complications; however, the potential risk of thromboembolism does exist. In addition, if all embolization materials fail, then a deconstructive procedure of the parent vessel is a last resort. The safety of this procedure lies in the results of a temporary balloon-occlusion test. In cases in which the patient fails the TBO test, a bypass procedure must be performed before the parent vessel is occluded. ${ }^{11-13}$

In the last decade, covered stents have been widely used within the aorta and peripheral, visceral, and coronary arteries as a safe and effective alternative to open surgical repair. ${ }^{17-21}$ Recently, covered stents have been used for the treatment of aneurysms, pseudoaneurysms, arterial dissections, and arteriovenous fistulas as well. ${ }^{2,5-8,22-26}$ Although several varieties of covered stents have been used by the neurovascular community, only the Willis covered stent (MicroPort, Shanghai, China) reported by Li et $\mathrm{al}^{22}$ is specially designed for intracranial vasculature use. 

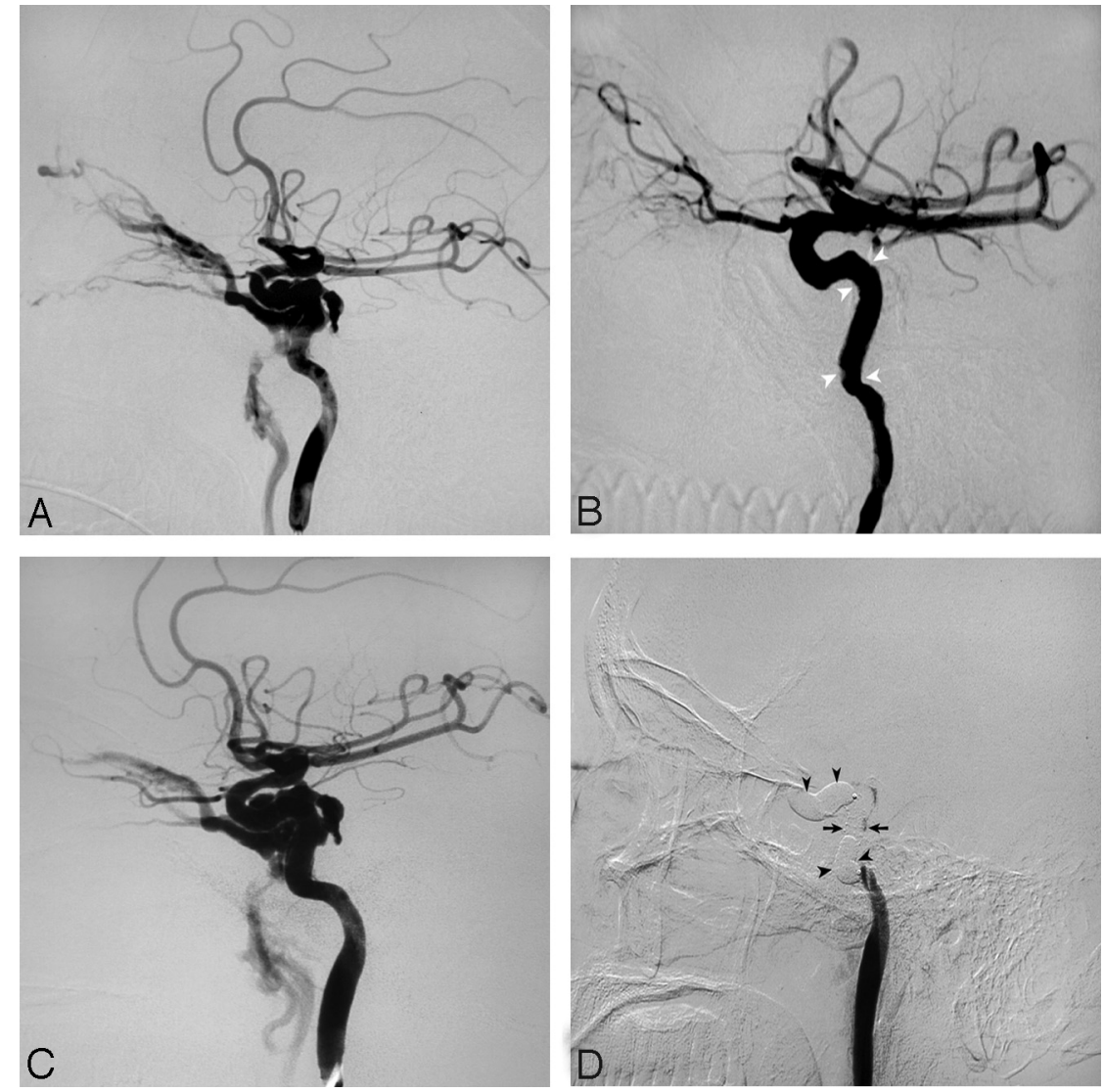

Fig 3. Case 7. $A$, Left carotid angiogram shows a DCCF. $B$, Immediate angiogram after covered-stent deployment and re-dilation shows complete occlusion of the fistula. Note the spasm at both ends of the stent (arrowheads). $C$, Angiogram 1 day after stent deployment shows recanalization of the fistula. D, The ipsilateral ICA was occluded with detachable balloons (arrowheads) at both ends of the stent (arrows).

The Jostent GraftMaster Coronary Stent Graft used in this series is composed of a sandwich construction of 2 bare stainless steel stents with a thin layer of polytetrafluoroethylene (PTFE) in between. Due to this composition and construction, the stent is rigid and cannot be navigated within tortuous anatomy. Possible complications that may result from this rigidity are dissection and periprocedural spasm of the cerebral arteries. However, if necessary precautions are taken both in patient selection and during the procedure by using necessary interventional adjunctive tools as described, technical success can be achieved with no harm to the patient.

Endoleak occurred in 3 patients (cases 3, 6, and 7) in this series. Re-dilation with a balloon of larger diameter resulted in transient exclusion (case 7), permanent exclusion (case 3), and persistent fistula in 1 patient (case 6). Archondakis et al ${ }^{2}$ reported 3 cases of endoleak as well in their treatment of 8 cases of traumatic carotid cavernous fistulas with covered stents. The presumed hypothesis is that though the ICA diameter is usually $<5 \mathrm{~mm}$, it can enlarge under long-standing high-flow conditions. Given the fact that the largest Jostent GraftMaster Coronary Stent Graft available is $5 \mathrm{~mm}$ in diameter, a parent vessel of a diameter $>5 \mathrm{~mm}$ will lead to an endoleak. This scenario occurred in 3 of our patients and certainly represents a roadblock in the use of covered stents within the intracranial vasculature. In 1 of these patients (case 7), the symptoms improved; however, they recurred the next morning. Cerebral angiography revealed an endoleak, thus resulting in sacrifice of the ICA.
A similar event was described by Archondakis et al. ${ }^{2}$ In 1 of their patients, cerebral angiography after stent placement showed complete exclusion of the fistula; however, an endoleak was discovered the next day. A plausible explanation for this lies in the fact that at the time of the procedure, the parent vessel is usually in spasm, thus creating a false impression of exclusion of the fistula. However, as the spasm resolves, a mismatch in size between the stent and parent vessel occurs, thus allowing an endoleak. Oversizing the stents in these situations could minimize such occurrences. Another plausible explanation for our patient (case 7) is the improper place at which the stent was deployed. Maybe the stent should have straightened out and covered the C3-4 segments of the ICA, thereby ensuring that the fistula was trapped between the ends of the stent. However, it failed to straighten out the C4 segment. Of course, the endoleak may also be caused by mismatch of the sectional shape, rupture of the PTFE, the presence of the "external" stent in the double-stent design, and possibly others. An oversized stent is preferred to prevent endoleaks in the first place, and multiple control angiograms are warranted to confirm its correct position before deployment.

In our group, all patients were premedicated with oral aspirin and clopidogrel and systematically heparinized during the procedure; then low-molecular heparin was given subcutaneously for 72 hours, followed by oral aspirin and clopidogrel. No thromboembolic events occurred after stent placement or during the follow-up period. Patients with traumatic DCCFs underwent a stent placement procedure at least 3 
months after their injury. Care must be taken in the use of anticoagulation and antiplatelet agents in those patients with hemorrhage or a tendency of hemorrhage, and those who were recently injured. Thromboembolism postprocedure is uncommon as evident in our and other series. Review of the literature revealed only 2 reported patients in whom the parent vessel was occluded on follow-up cerebral angiography. Both patients were asymptomatic, and the occlusion was found on routine follow-up. In 1 patient, occlusion of the ICA was found 1 week after stent placement for a posttraumatic petrocavernous ICA pseudoaneurysm. This patient also had a distal MCA pseudoaneurysm; thus minimal anticoagulation was used (aspirin daily). ${ }^{5}$ Another case of ICA occlusion was found at the 1-month angiographic follow-up, which was thought to be the result of discontinuation of the antiplatelet therapy. ${ }^{2}$ Archondakis et $\mathrm{al}^{2}$ reported an asymptomatic 30\% intrastent stenosis at 6-month follow-up angiography, which improved at the 1-year follow-up.

Covered stents by definition have the pitfall of occluding small perforating vessels in the region in which the stent has been deployed. Although the cavernous segment does have branches, to our knowledge, the clinical significance of occluding these branches is not well reported in the literature. Most certainly this is a reason covered stents cannot be used in all segments of the intracranial circulation. However, the use of covered stents in the cavernous segment is well tolerated by patients as documented in the literature and our own series.

\section{Conclusions}

Our experience suggests that covered-stent placement represents a reasonable alternative to detachable balloons or coils, which have mass effect on the cavernous sinus. Larger sample sizes with longer duration of follow-up is required to evaluate the efficacy of covered stents versus balloons or coils.

\section{Acknowledgments}

We sincerely thank Aditya S. Pandey, MD, from Thomas Jefferson University Hospital, for his kindness in revising the language during preparation of the manuscript.

\section{References}

1. Barrow DL, Spector RH, Braun IF, et al. Classification and treatment of spontaneous carotid-cavernous sinus fistulas. J Neurosurg 1985;62:248-56

2. Archondakis E, Pero G, Valvassori L, et al. Angiographic follow-up of traumatic carotid cavernous fistulas treated with endovascular stent graft placement. AJNR Am J Neuroradiol 2007;28:342-47

3. Rooij WJ, Sluzewski M, Beute GN. Ruptured cavernous sinus aneurysms causing carotid cavernous fistula: incidence, clinical presentation, treatment, and outcome. AJNR Am J Neuroradiol 2006;27:185-89
4. Kobayashi N, Miyachi S, Negoro M, et al. Endovascular treatment strategy for direct carotid-cavernous fistulas resulting from rupture of intracavernous carotid aneurysms. AJNR Am J Neuroradiol 2003;24:1789-96

5. Redekop G, Marotta T, Weill A. Treatment of traumatic aneurysms and arteriovenous fistulas of the skull base by using endovascular stents. J Neurosurg 2001;95:412-19

6. Kocer N, Kizilkilic O, Albayram S, et al. Treatment of iatrogenic internal carotid artery laceration and carotid cavernous fistula with endovascular stentgraft placement. AJNR Am J Neuroradiol 2002;23:1589-95

7. Felber S, Henkes H, Weber W, et al. Treatment of extracranial and intracranial aneurysms and arteriovenous fistulae using stent grafts. Neurosurgery 2004;55:459-62

8. Luo CB, Teng MM, Yen DH, et al. Endovascular embolization of recurrent traumatic carotid-cavernous fistulas managed previously with detachable balloons. J Trauma 2004;56:1214-20

9. Parkinson D. Carotid cavernous fistula: direct repair with preservation of the carotid artery: technical note. J Neurosurg 1973;38:99-106

10. Serbinenko FA. Balloon catheterization and occlusion of major cerebral vessels. J Neurosurg 1974;41:125-45

11. Gupta AK, Purkayastha S, Krishnamoorthy T, et al. Endovascular treatment of direct carotid cavernous fistulae: a pictorial review. Neuroradiology 2006;48:831-39

12. Lewis AI, Tomsick TA, Tew JM Jr. Management of $\mathbf{1 0 0}$ consecutive direct carotid-cavernous fistulas: results of treatment with detachable balloons. $\mathrm{NeU}$ rosurgery 1995;36:239-45

13. Debrun G, Lacour P, Vinuela F, et al. Treatment of $\mathbf{5 4}$ traumatic carotid-cavernous fistulas. J Neurosurg 1981;55:678-92

14. Klisch J, Schipper J, Husstedt $\mathrm{H}$, et al. Trans-sphenoidal computer-navigationassisted deflation of a balloon after endovascular occlusion of a direct carotid cavernous sinus fistula. AJNR Am J Neuroradiol 2001;22:537-40

15. Ahn JY, Lee BH, Joo JY. Stent-assisted Guglielmi detachable coil embolisation for the treatment of a traumatic carotid cavernous fistula. J Clin Neurosci 2003; 10:96-98

16. Luo CB, Teng MM, Chang FC, et al. Transarterial balloon-assisted n-butyl-2cyanoacrylate embolization of direct carotid cavernous fistulas. AJNR Am J Neuroradiol 2006;27:1535-40

17. Dake MD, Miller DC, Semba CP, et al. Transluminal placement of endovascular stent-grafts for the treatment of descending thoracic aortic aneurysms. N Engl J Med 1994;331:1729-34

18. Köcher M, Utíkal P, Koutná J, et al. Endovascular treatment of abdominal aortic aneurysms: 6 years of experience with Ella stent-graft system. Eur J Radiol 2004;51:181-88

19. Bartorelli AL, Trabattoni D, Reali M. Percutaneous obliteration of an iatrogenic pseudoaneurysm of the right subclavian artery with a PTFE-covered stent-graft. Int J Cardiovasc Intervent 2001;4:195-96

20. Ogino H, Banno T, Sato Y, et al. Superior mesenteric artery stent-graft placement in a patient with pseudoaneurysm developing from a pancreatic pseudocyst. Cardiovasc Intervent Radiol 2004;27:68-70

21. Briguori C, Sarais C, Sivieri G, et al. Polytetrafluoroethylene-covered stent and coronary artery aneurysms. Catheter Cardiovasc Interv 2002;55:326-30

22. Li MH, Li YD, Gao BL, et al. A new covered stent designed for intracranial vasculature: application in the management of pseudoaneurysm of the cranial internal carotid artery. AJNR Am J Neuroradiol 2007;28:1579-85

23. Cohen JE, Grigoriadis S, Gomori JM. Petrous carotid artery pseudoaneurysm in bilateral carotid fibromuscular dysplasia: treatment by means of self-expanding covered stent. Surg Neurol 2007;68:216-20

24. Saatci I, Cekirge HS, Ozturk MH, et al. Treatment of internal carotid artery aneurysms with a covered stent: experience in 24 patients with mid-term follow-up results. AJNR Am J Neuroradiol 2004;25:1742-49

25. Schönholz C, Krajcer Z, Carlos Parodi J, et al. Stent-graft treatment of pseudoaneurysms and arteriovenous fistulae in the carotid artery. Vascular 2006;14:123-29

26. Blasco J, Macho JM, Burrel M, et al. Endovascular treatment of a giant intracranial aneurysm with a stent-graft. J Vasc Interv Radiol 2004;15:1145-49 